\title{
A Practical Method to Design Reflector-Based Light-Emitting Diode Luminaire for General Lighting
}

\author{
Jinren Yan \\ Department of Optoelectronics and Communication Engineering, \\ Yunnan Open University (Yunnan Vocational \& Technical College of National Defense Industry), Xuefu Road 113, \\ Kunming 650223, China \\ Correspondence should be addressed to Jinren Yan; renyj0720@163.com
}

Received 2 August 2018; Accepted 3 October 2018; Published 18 October 2018

Academic Editor: Somenath N. Sarkar

Copyright (C) 2018 Jinren Yan. This is an open access article distributed under the Creative Commons Attribution License, which permits unrestricted use, distribution, and reproduction in any medium, provided the original work is properly cited.

\begin{abstract}
A reflector-based light-emitting diode (LED) luminaire structure that can achieve a large cut-off angle for general lighting is presented in this work. The proposed lighting unit mainly consists of a spherical reflector and a primary packaging lens that contains an aspheric surface and a spherical surface. The light rays emitted from the LED light source are well controlled by the spherical reflector and the aspheric surface of the lens for the purpose of obtaining a uniform illumination on the target surface. Both the ideal Lambertian LED and non-Lambertian LED light sources were employed to validate the proposed structure and the performance of the designed lighting units was analyzed by optical simulation. The results show that the light utilization efficiencies and the estimated uniformities are $92.96 \%$ and $91.11 \%$ for ideal Lambertian LED-based lighting unit and $93.31 \%$ and $91.64 \%$ for nonLambertian LED-based lighting unit, respectively. Further analysis shows that the tolerances of horizontal, vertical, and rotational deviation of the both lighting units were about $2.0 \mathrm{~mm}, 1.0 \mathrm{~mm}$, and $1.0^{\circ}$, respectively.
\end{abstract}

\section{Introduction}

After years of development, light-emitting diodes (LEDs) have been widely used in the fields of indoor lighting, road lighting, automobile headlights, backlight of liquid crystal display, and so forth [1-4]. Different from the conventional light sources, such as the incandescent lamp and the fluorescent lamp, the LED light source is a Lambertian-like emitter and the light intensity distribution curve (LIDC) follows a cosine power function [5]. Thus, a secondary optical element is generally needed to redistribute the light energy emitted from LED to meet the specific illumination requirements. Over the past decade, the freeform lenses and the freeform reflectors have been widely used in the design of LED secondary optics in illumination designs [5-10]. In real applications, especially in outdoor lighting, the reflector is considered as an important optical component which can not only be used to modulate the light energy emitted from the light source to achieve specific lighting, but also be employed as the protector of the lighting equipment. However, it is still a challenge to faultlessly control all of the light rays emitted from the LED chip by a single freeform reflector alone. The optimal cut-off angle of the reflector is limited by the LIDC of the LED light source for the case of uniform lighting [11]. For example, for an ideal Lambertian LED, the largest cut-off angle of the freeform reflector is about $45^{\circ}$ for uniform illumination [12]. As the cut-off angle of the reflector increased larger than $45^{\circ}$, the uniformity will deteriorate. Such restriction put up some obstacles in using LEDs in the field of general lighting where the cut-off angle of the lighting unit typically defined between $55^{\circ}$ and $65^{\circ}[12,13]$.

In this work, a reflector-based LED luminaire structure which can achieve a large cut-off angle for general lighting is developed and the method for the design of the developed lighting unit structure is presented in detail. The structure of the lighting unit only consists of a spherical reflector and a primary lens which comprises an aspheric surface and a spherical surface. In the proposed lighting unit, the reflector is employed to modulate the side rays with an emitting angle larger than the specified cut-off angle of the lighting unit and 


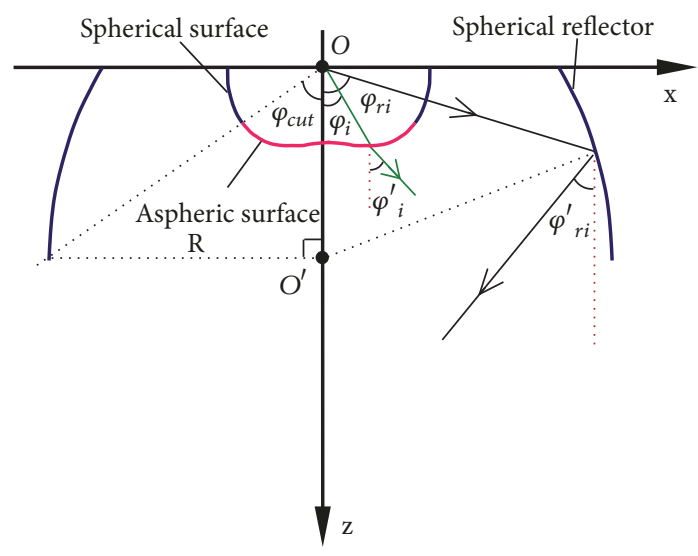

FIGURE 1: Geometrical diagram of the developed LED lighting unit.

the aspheric surface of the primary lens is used to modulate the light rays with an emitting angle smaller than the cut-off angle. Then the uniform illumination on the target surface is formed by superposition of the light energy reflected by the spherical reflector and the light energy modulated by the aspheric surface of the lens.

\section{Design Method}

In order to obtain a uniform far-field illumination on the target surface, the LIDC of the lighting equipment should comply with the following expression [14]:

$$
I_{l s}(\varphi)=\frac{I_{l 0}}{\cos ^{3}(\varphi)}
$$

where $I_{l s}(\varphi)$ represents the luminous intensity distribution of the lighting equipment and $I_{l 0}$ is the luminous intensity at $\varphi=0^{\circ}$. Figure 1 shows the geometrical structure of the developed lighting unit. As depicted in Figure 1, the proposed structure comprises a spherical reflector and a primary lens. One can note that the central part of the lens is an aspheric surface and the side part of the lens is a spherical surface with the center located at the origin $O$ of the coordinates. The LED light source is immersed into the primary lens and also positioned at the origin $O$ of the coordinates [15-17], $O^{\prime}$ is the center of the spherical reflector, and $R$ denotes the radius of the reflector. As can be noted from Figure 1, the opening dimension of the spherical reflector equals its diameter. $\varphi_{c u t}$ represents the cut-off angle of the lighting unit and it is generally given by designers. $\varphi_{i}$ denotes the angle between the light ray that will be refracted by the aspheric surface of the lens and z-axis, and $\varphi_{i}^{\prime}$ is the angle between the corresponding refracted light ray and $\mathrm{z}$-axis. One can note that since the side part of the lens is spherical surface, the light rays will directly pass through the spherical surface of the lens without deflection. Thus, $\varphi_{r i}$ denotes the angle between the light ray that will be reflected by the spherical reflector and $\mathrm{z}$-axis, and $\varphi_{r i}^{\prime}$ is the angle between the corresponding reflected light ray and $\mathrm{z}$-axis.

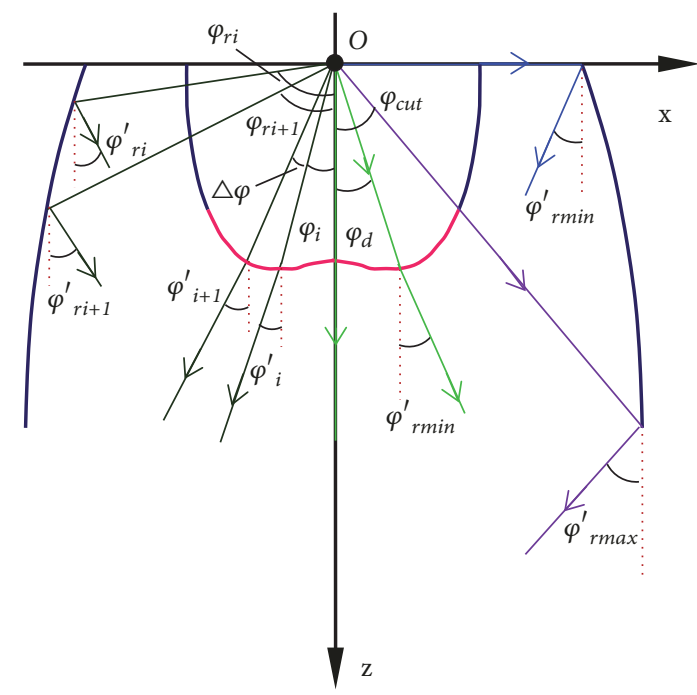

FIGURE 2: Diagrammatic sketch of the light energy distribution of the lighting unit.

From the geometrical relationship illustrated in Figure 1, the relationship between the light reflection angle $\varphi_{r i}^{\prime}$ and the light-emitting angle $\varphi_{r i}$ of the side rays can be written as

$$
\varphi_{r i}^{\prime}=\pi-\varphi_{r i}-2 \operatorname{acrsin}\left[\frac{\sin \left(\varphi_{r i}\right)}{\tan \left(\varphi_{c u t}\right)}\right]
$$

As illustrated in Figure 1, the light-emitting angle $\varphi_{r i}$ ranges from $\varphi_{\text {cut }}$ to $\pi / 2$. Consider the reflection characteristic of the spherical reflector in this case, the minimum value $\varphi_{\text {rmin }}^{\prime}$ and the maximum value $\varphi_{\text {rmax }}^{\prime}$ of the light reflection angle $\varphi_{r i}^{\prime}$ can be obtained when the light-emitting angle $\varphi_{r i}$ reaches $\pi / 2$ and $\varphi_{c u t}$, respectively. Since the reflection angle of the light ray reflected by the spherical reflector is invariant for a specified emitting angle in this case, the uniform lighting on the target surface should be achieved by redistributing the light energy passing through the aspheric surface of the lens. The right side of Figure 2 shows the diagrammatic sketch of the light energy distribution of the developed lighting unit. According to the geometrical relationship shown in Figure 2, one can note that the light reflection angle $\varphi_{r \max }^{\prime}$ equals $\varphi_{\text {cut }}$. As shown in Figure 2, the light energy emitted from the LED light source can be divided into three parts: the first part contains the light energy in the angle range of $0^{\circ}$ to $\varphi_{d}$ and the light energy in this part is refracted to the angle range of $0^{\circ}$ to $\varphi_{\text {rmin }}^{\prime}$ by the aspheric surface of the lens; the second part contains the light energy in the angle range of $\varphi_{d}$ to $\varphi_{c u t}$ and the third part contains the light energy in the angle range of $\varphi_{c u t}$ to $\pi / 2$. The light energy in these two parts is modulated to the angle range of $\varphi_{\text {rmin }}^{\prime}$ to $\varphi_{\text {rmax }}^{\prime}$ by the aspheric surface of the lens and the spherical reflector, respectively. 
Based on the contents aforementioned and the law of energy conservation, the following two equations can be obtained, respectively:

$$
\begin{aligned}
& 2 \pi \int_{0}^{\varphi_{d}} I_{s}(\varphi) \sin (\varphi) d \varphi=2 \pi \int_{0}^{\varphi^{\prime}{ }_{\text {min }}} I_{l s}(\varphi) \sin (\varphi) d \varphi \\
& 2 \pi \int_{\varphi_{d}}^{\varphi_{c u t}} I_{s}(\varphi) \sin (\varphi) d \varphi+2 \pi \rho \int_{\varphi_{c u t}}^{\pi / 2} I_{s}(\varphi) \sin (\varphi) d \varphi \\
& \quad=2 \pi \int_{\varphi^{\prime}{ }_{\text {min }}}^{\varphi_{\text {cut }}} I_{l s}(\varphi) \sin (\varphi) d \varphi
\end{aligned}
$$

where $\rho$ denotes the reflectivity of the reflector; in this work, it is supposed to be $95 \% . I_{s}(\varphi)$ represents the luminous intensity distribution of the LED light source. $I_{l s}(\varphi)$ represents the luminous intensity distribution of the lighting unit as it is expressed by (1).

To construct the aspheric surface of the lens, the relationship between the light-emitting angle $\varphi_{i}$ and the corresponding refraction angle $\varphi^{\prime}{ }_{i}$ should be obtained at first, as shown in the left side of Figure 2. It is noted that $\triangle \varphi$ shown in the figure represents the preset angle increment of the incident rays and it is set as $0.25^{\circ}$ in this work. Then, $\varphi_{i}=i \Delta \varphi$ $(i=0,1,2, \ldots, N)$ can be obtained easily. Using the law of energy conservation and the edge-ray principle [18], the following two equations are obtained, respectively:

$$
\begin{aligned}
& 2 \pi \int_{0}^{\varphi_{i}} I_{s}(\varphi) \sin (\varphi) d \varphi=2 \pi \int_{0}^{\varphi^{\prime}} I_{l s}(\varphi) \sin (\varphi) d \varphi \\
& 2 \pi \int_{\varphi_{d}}^{\varphi_{i}} I_{s}(\varphi) \sin (\varphi) d \varphi+2 \pi \rho \int_{\varphi_{r i}}^{\pi / 2} I_{s}(\varphi) \sin (\varphi) d \varphi \\
& \quad=2 \pi \int_{\varphi^{\prime}{ }_{r \min }}^{\varphi^{\prime}{ }_{i}} I_{l s}(\varphi) \sin (\varphi) d \varphi
\end{aligned}
$$

It is worth noting that (6) implies the condition that $\varphi^{\prime}{ }_{i}$ equals $\varphi_{r i}^{\prime}$. Thus (2) could be substituted into (6) to replace $\varphi_{i}^{\prime}$. Substituting $\varphi_{i}$ into (5) and (6), respectively, the corresponding $\varphi^{\prime}{ }_{i}$ for the angle range of $0^{\circ}$ to $\varphi_{d}$ and the angle range of $\varphi_{d}$ to $\varphi_{c u t}$ could be obtained. After obtaining the relationships between $\varphi_{i}$ and $\varphi_{i}^{\prime}$, the aspheric surface of the primary lens can be easily constructed using the method described in [15].

\section{Design Examples}

Using the method described in Section 2, an LED lighting unit with a cut-off angle of $\varphi_{c u t}=60^{\circ}$ was designed as an example. Substituting $\pi / 2$ and $\varphi_{c u t}$ into (2), the minimum angle $\varphi_{r \text { min }}^{\prime}$ and maximum angle $\varphi_{\text {rmax }}^{\prime}$ of the reflected light rays are calculated as $19.5^{\circ}$ and $60^{\circ}$, respectively. In the simulation, it is assumed that the LED light source has a dimension of $1.0 \mathrm{~mm} \times 1.0 \mathrm{~mm}$. The material of the lens is assumed to be PMMA (Polymethyl Methacrylate) with a refractive index of $n=1.49$. It is assumed that the LED light source emits $100 \mathrm{~lm}$ light energy and 1.0 million rays were used for simulation. The lighting unit is $5 \mathrm{~m}$ away from the target surface.
3.1. Design with Ideal Lambertian LED. For an ideal Lambertian LED, the LIDC of the light source can be expressed as

$$
I_{s}(\varphi)=I_{0} \cos (\varphi)
$$

where $I_{0}$ denotes the luminous intensity at $\varphi=0^{\circ}$. Substituting (1) and (7) into (3) and (4), the parameter $\varphi_{d}$ and the ratio of $I_{0}$ to $I_{l 0}$ can be attained:

$$
\begin{aligned}
& \varphi_{d}=\arcsin \left[\frac{\tan \left(\varphi_{r \text { min }}^{\prime}\right)}{\tan \left(\varphi_{\text {cut }}\right)} \sqrt{(1-\rho) \sin ^{2}\left(\varphi_{\text {cut }}\right)+\rho}\right] \\
& \frac{I_{0}}{I_{l 0}}=\frac{\tan ^{2}\left(\varphi_{r \text { min }}^{\prime}\right)}{\sin ^{2}\left(\varphi_{d}\right)}
\end{aligned}
$$

Using (8) and (9), the value of parameter $\varphi_{d}$ and the ratio of $I_{0}$ to $I_{l 0}$ are about $11.6^{\circ}$ and 3.08 in this case. Then, substituting (1), (2), (7), (8), and (9) into (5) and (6), one can find the following two expressions, respectively:

$$
\begin{gathered}
\varphi_{i}^{\prime}=\arctan \left(\sqrt{\frac{I_{0}}{I_{l 0}} \sin ^{2}\left(\varphi_{i}\right)}\right) \quad \varphi_{i} \in\left[0, \varphi_{d}\right] \\
\frac{I_{0}}{I_{l 0}}\left[\sin ^{2}\left(\varphi_{i}\right)-\sin ^{2}\left(\varphi_{d}\right)+\rho\left(1-\sin ^{2}\left(\varphi_{r i}\right)\right)\right] \\
-\tan ^{2}\left(\varphi_{r i}+2 \arcsin \left(\frac{\sin \left(\varphi_{r i}\right)}{\tan \left(\varphi_{\text {cut }}\right)}\right)\right. \\
\left.\quad+\tan ^{2}\left(\varphi_{r \text { min }}^{\prime}\right)\right)=0 \quad \varphi_{i} \in\left(\varphi_{d}, \varphi_{\text {cut }}\right]
\end{gathered}
$$

It can be seen that, for the angle range of $0^{\circ}$ to $\varphi_{d}, \varphi^{\prime}{ }_{i}$ can be obtained easily by iteratively substituting $\varphi_{i}$ into (10). However, the relationship between $\varphi_{i}$ and $\varphi^{\prime}{ }_{i}$ in the angle range of $\varphi_{d}$ to $\varphi_{c u t}$ is expressed by (11), which is a nonlinear equation and is solved by using numerical method [19]. It should be emphasized that solving (11) can only get the relationship between $\varphi_{i}$ and $\varphi_{r i}$, the corresponding refraction angle $\varphi^{\prime}{ }_{i}$ of $\varphi_{i}$ should be obtained by substituting $\varphi_{r i}$ into (2).

Figure 3 shows the calculated geometrical profile of the proposed lighting unit for the ideal Lambertian LED light source and the corresponding geometrical model. The simulation results of the developed lighting unit are shown in Figure 4. Figure 4(a) shows the irradiance map of the developed lighting unit. It can be seen that a uniform illumination is obtained on the target surface. The dot line in the figure represents the slicing position of the slicing chart. Figure 4(b) shows the light intensity distribution of the ideal Lambertian LED and the designed lighting unit; it can be seen that the intensity peak of the designed lighting unit is located at about $\pm 58^{\circ}$ with the light energy sheared almost at $\pm 60^{\circ}$; the simulation results are in good agreement with expectations.

The light utilization efficiency, which is defined as the ratio of the light energy within the circle region with the radius of $8660 \mathrm{~mm}$ to the light energy emitted from the LED light source, is about $92.96 \%$. The illuminance uniformity, which is defined as the ratio of the minimum illuminance to the average illuminance $\left(U=E_{\text {min }} / E_{\text {ave }}\right)$ on the target region, is estimated as $91.11 \%$. 


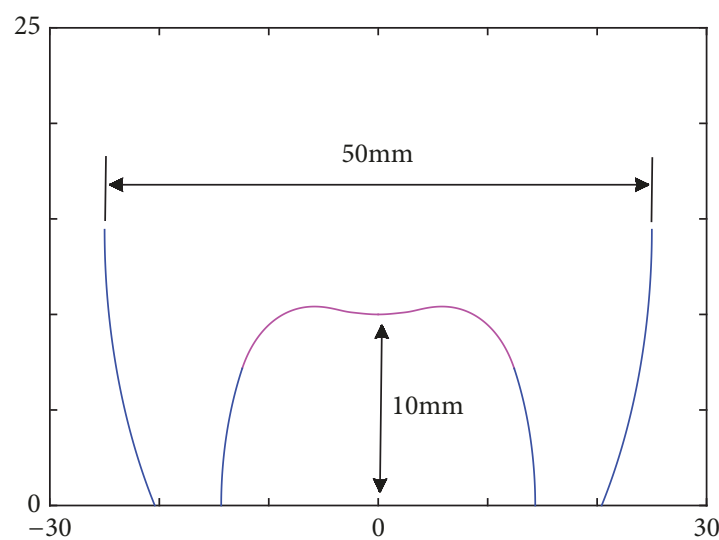

(a)

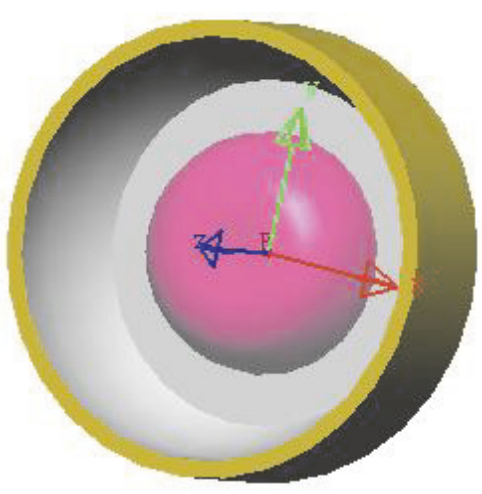

(b)

FIgUre 3: (a) Geometrical profile of the designed lighting unit for the ideal Lambertian LED light source and (b) the corresponding geometrical model.

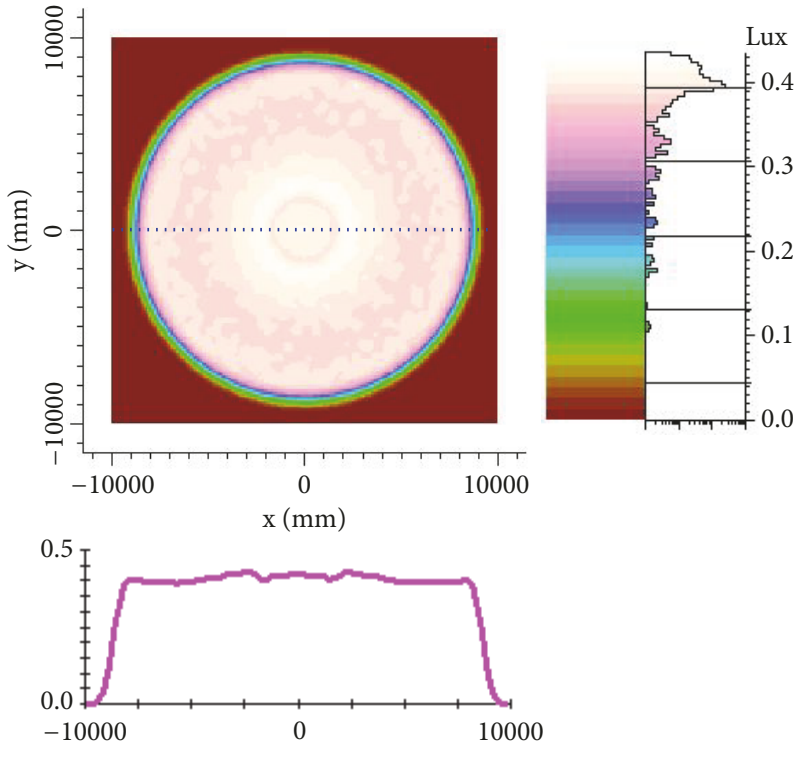

(a)

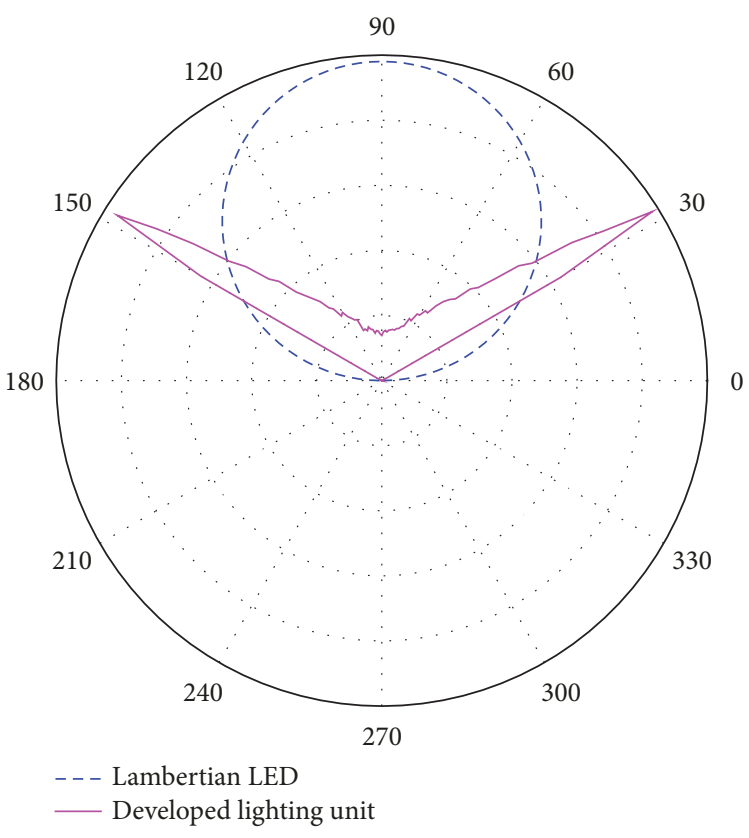

(b)

FIGURE 4: (a) Irradiance map and (b) the angular intensity distribution of the developed lighting unit for the ideal Lambertian LED.

3.2. Design with Non-Lambertian LED. Figure (5) shows the LIDC of a non-Lambertian LED light source [13]. As shown in the figure, the LIDC of the LED cannot be simply expressed by a simple cosine power function. In general, the LIDCs of the non-Lambertian LED light sources can be fitted by a polynomial function [20], Gaussian function, or cosine function [21]. In this study, for the purpose of simple, the polynomial function was employed to represent the LIDC of the non-Lambertian LED light source:

$$
I_{s}(\varphi)=I_{0}\left(a_{0}+a_{1} \varphi^{2}+a_{2} \varphi^{4}+a_{3} \varphi^{6}+a_{4} \varphi^{8}\right)
$$

where $a_{i}(i=0,1,2,3,4)$ represent the coefficients of the polynominal function. $I_{0}$ is the luminous intensity at $\varphi=0^{\circ}$. After curve fitting, the coefficients of the polynomial function used to represent the LIDC of the non-Lambertian LED showed in Figure 5 are shown in Table 1, and the attained LIDC model is shown in Figure 5.

Substituting (1) and (12) into (3) and (4), the value of parameter $\varphi_{d}$ and the ratio of $I_{0}$ to $I_{l 0}$ can be obtained; the obtained results are shown in Table 1 . Then these two attained parameter values and (1) and (12) are substituted into (5) and (6), respectively; the relationship between the lighting emitting angle $\varphi_{i}$ and the corresponding refraction angle $\varphi_{i}^{\prime}$ can be obtained. It is worth noting that substituting (12) into (4), (5), and (6) will lead to nonlinear equations; thus, the numerical method should be employed to solve these [19].

Figure 6 shows the calculated profile of the lighting unit for the non-Lambertian LED light source and the 


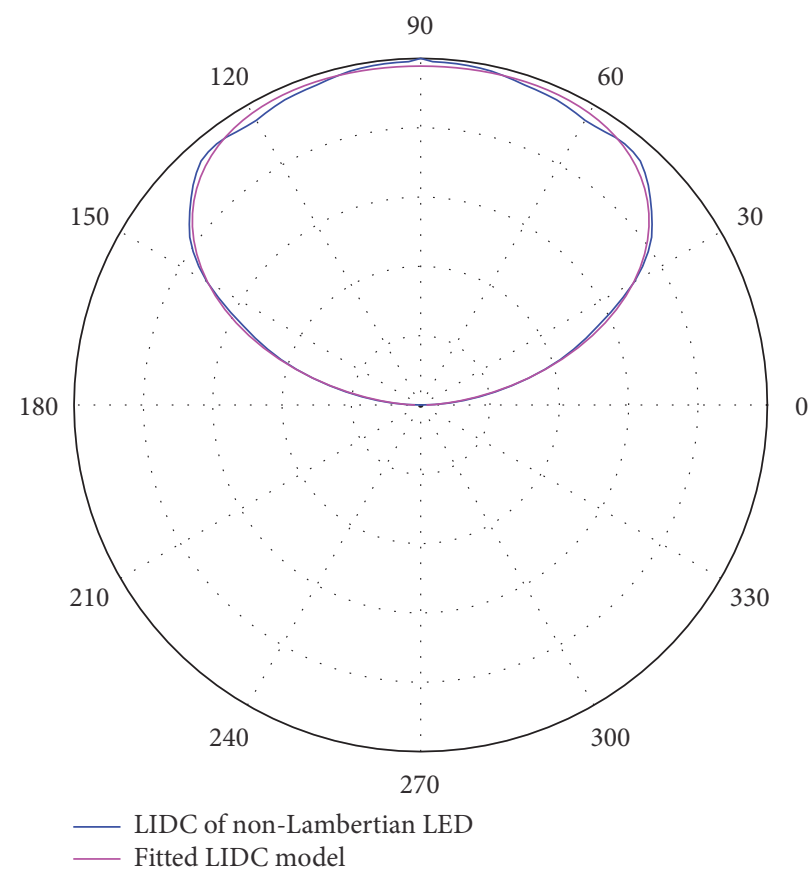

FIGURE 5: The LIDC of a non-Lambertian LED light source and the corresponding model fitted by a polynomial function.

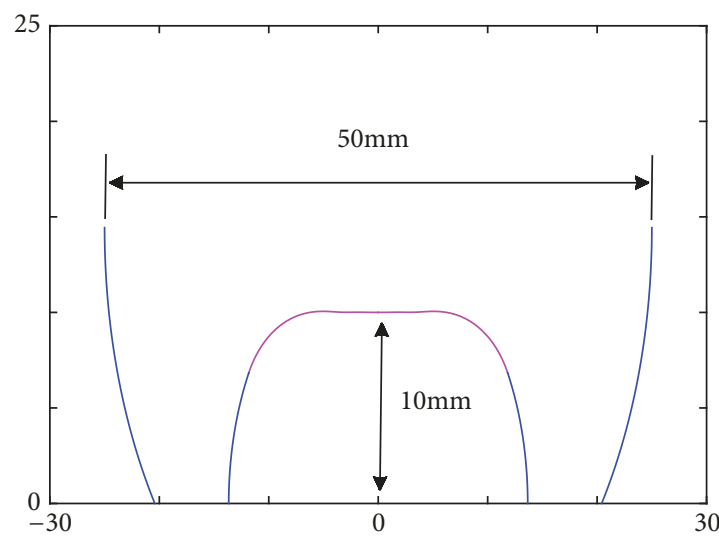

(a)

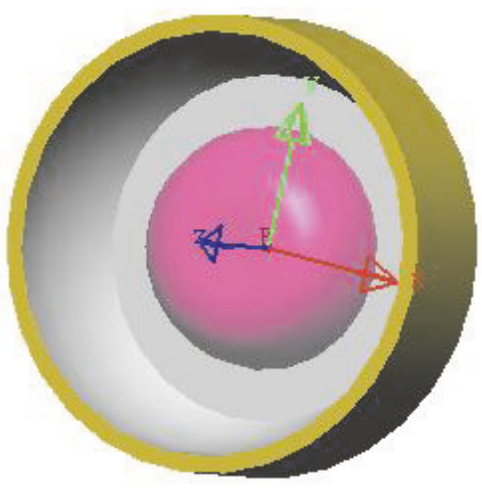

(b)

FIGURE 6: (a) Geometrical profile of the non-Lambertian LED-based luminaire and (b) the corresponding three dimensional model.

TABLE 1: The coefficients of the polynomial function, the ratio of $I_{0}$ to $I_{l 0}$, and the value of parameter $\varphi_{d}$.

\begin{tabular}{lccc}
\hline Parameter & $\boldsymbol{a}_{\boldsymbol{i}}$ & $\boldsymbol{I}_{\mathbf{0}} / \boldsymbol{I}_{\mathbf{l 0}}$ & $\boldsymbol{\varphi}_{\boldsymbol{d}}$ \\
\hline \multirow{3}{*}{ Value } & $\mathrm{a}_{0}=0.9778 ; \mathrm{a}_{1}=0.05183$ & & \\
& $\mathrm{a}_{2}=-0.2492 ; \mathrm{a}_{3}=-0.05671$ & 4.89 & $13^{\circ}$ \\
& $\mathrm{a}_{4}=0.0346$ & & \\
\hline
\end{tabular}

corresponding geometrical model. The simulation results of the non-Lambertian LED-based lighting unit are shown in Figure 7. Figure 7(a) shows the irradiance map of the developed lighting unit. One can note that a uniform illumination is obtained on the target surface. Figure $7(\mathrm{~b})$ shows the angular intensity distribution of the non-Lambertian LED and the designed lighting unit, one can note that the intensity peak of the designed lighting unit is also located at about $\pm 58^{\circ}$ with the light energy sheared almost at $\pm 60^{\circ}$. The light utilization efficiency and the estimated uniformity of the designed unit are about $93.31 \%$ and $91.64 \%$, respectively.

3.3. Tolerance Analysis. The installation error is one of the most important issues for optical system because the optical performance of the optical system will be deteriorated by position deviation of the components. In this work, the tolerance analysis will be focused on discussing the position migration and rotation of the spherical reflector. Figures 8(a), 9(a), and 10(a) show three possible installation errors of the developed two lighting units in real applications, which represent the position migrations of the reflector in 


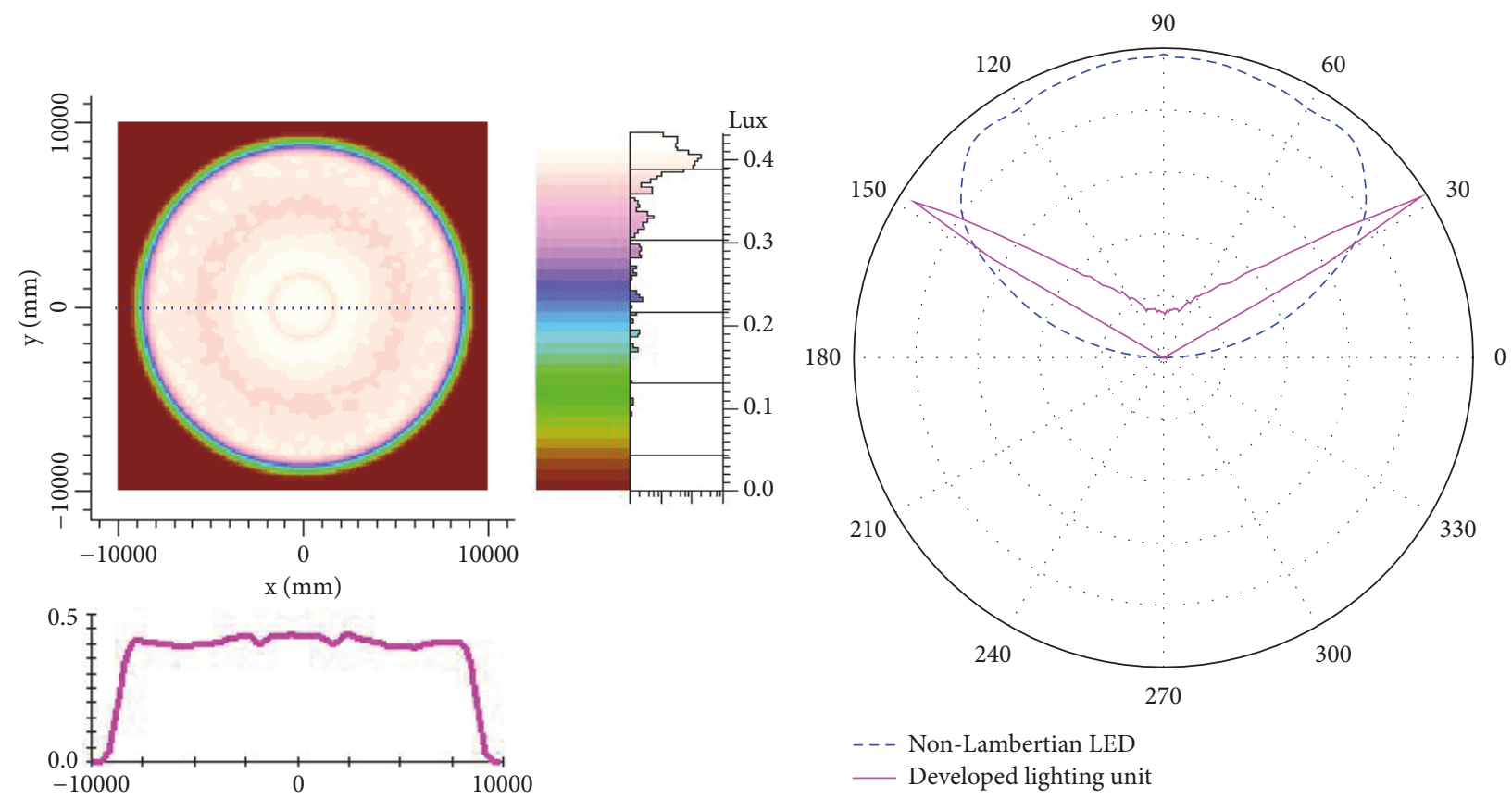

(a)

(b)

FIGURE 7: (a) Irradiance map and (b) the angular intensity distribution of the lighting unit for non-Lambertian LED.

TABLE 2: The optical performance of the SFRB luminaires and the newly developed luminaires for both LED light sources.

\begin{tabular}{lccc}
\hline Structure & LED Type & Uniformity & Efficiency \\
\hline \multirow{2}{*}{ Single Reflector } & Lambertian LEd & $47.52 \%$ & $97.70 \%$ \\
& Non-Lambertian LEd & $56.63 \%$ & $97.35 \%$ \\
\hline \multirow{2}{*}{ Developed Structure } & Lambertian LEd & $91.11 \%$ & $92.96 \%$ \\
& Non-Lambertian LEd & $91.64 \%$ & $93.31 \%$ \\
\hline
\end{tabular}

horizontal direction (x-axis direction), vertical direction ( $\mathrm{z}$ axis direction), and rotational direction (rotate about $y$ axis), respectively. Figures 8(b), 9(b), and 10(b) show the corresponding effects on the light utilization efficiencies of the two lighting units and the uniformities on the target surfaces. One can note that these three installation errors have little influence on the light utilization efficiencies of the two lighting units. However, the uniformities on the target surfaces are deteriorated as long as any of these three installation errors increases. One can note that the installation deviation $\Delta \theta$ performs the highest sensitivity to the uniformities on the target surface and the deviation of $\Delta \mathrm{x}$ performs the lowest sensitivity. According to the lighting standards, such as GB 50034-2013 "Standard for lighting design of buildings" and EN 12464-1:2002 "Light and lighting-Lighting of work places," to meet most of general lighting applications, the illuminance uniformity on the target surface should be greater than $70 \%$. Thus, after checking the simulation results of the two lighting units, the uniformity on the target surface is better than $70 \%$ when the installation errors $\Delta x, \Delta z$, and $\Delta \theta$ less than or equal to $2.0 \mathrm{~mm}, 1.0 \mathrm{~mm}$, and $1.0^{\circ}$, respectively.
3.4. Discussion. As a comparison, the luminaires with a single freeform reflector and a bare LED light source were designed using the method described in [11]. Figures 11(a) and 11(b) show the geometrical profiles of the freeform reflectors for the ideal Lambertian LED and the non-Lambertian LED, respectively. Both of the freeform reflectors have a similar cross section; thus, only the geometrical model of the freeform reflector for the ideal Lambertian LED is showed in Figure 11(c).

Figures 12 and 13 show the simulation results of the single freeform reflector-based (SFRB) luminaires for the ideal Lambertian LED and the non-Lambertian LED. As shown in these two figures, the illuminance uniformities on the target region are deteriorated a lot compared with the newly developed LED luminaire structure. Table 2 shows the optical performances of the SFRB luminaires and the developed luminaires for both the ideal Lambertian LED and the nonLambertian LED light sources. One can note from the table that the light energy utilization efficiencies of the SFRB lighting units are better than the newly developed lighting units for both LED light sources; however, the uniformities are unacceptable for the SFRB lighting units. Due to Fresnel 


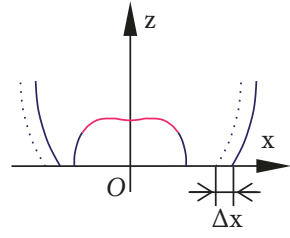

(a)

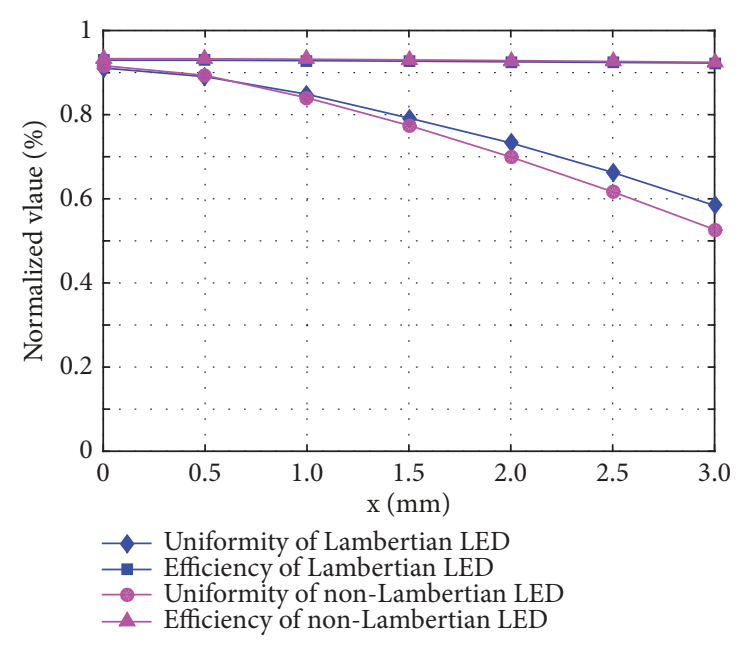

(b)

Figure 8: (a) Schematic of horizontal deviation $\Delta \mathrm{x}$ and (b) influence of horizontal deviation on the illumination uniformity and the light utilization efficiency.

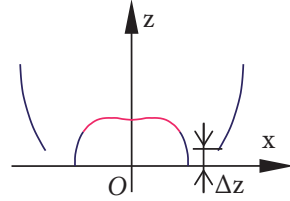

(a)

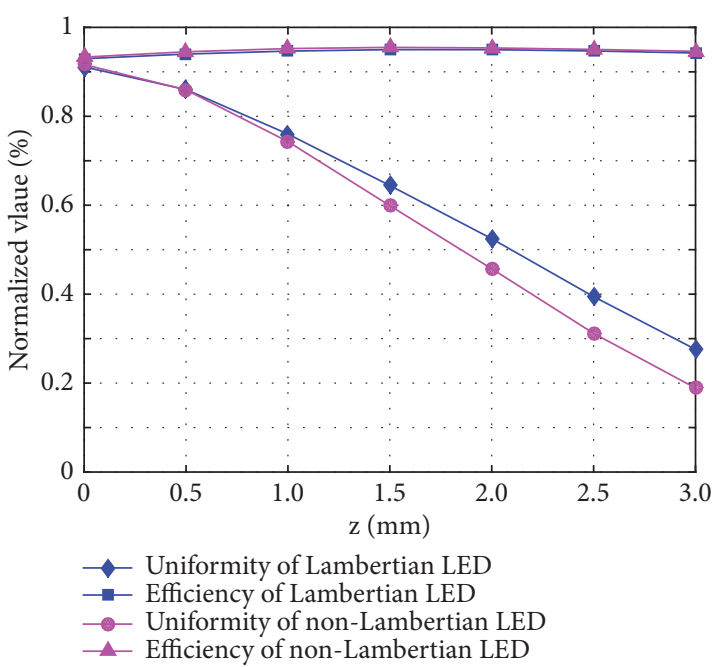

(b)

FIGURE 9: (a) Schematic of vertical deviation $\Delta \mathrm{z}$ and (b) influence of vertical deviation on the illumination uniformity and the light utilization efficiency.

reflection on the primary packaging lens surface, both of the developed lighting units suffers about $4 \%$ more light energy loss compared with the SFRB lighting luminaires, even so, the uniformities of the developed lighting units are much better than the SFRB lighting units.

\section{Conclusions}

A reflector-based LED lighting unit structure that can achieve a large cut-off angle for general lighting has been discussed in this work. The unit only consists of a spherical reflector and a primary packaging aspheric lens. All of the light rays emitted from LED light source can be well modulated by the reflector and the aspheric surface of the primary lens. The developed lighting unit structure overcomes the shortcomings that exist in the SFRB lighting unit in which the light energy close to the optical axis of the lighting system cannot be modulated by the freeform reflector and the illuminance uniformity will deteriorate when the cut-off angle of the reflector is larger than 45 . In addition, the freeform surface is only designed in the primary packaging lens which is widely used in LED encapsulation and the reflector is a spherical reflector; the manufacturing cost of the developed luminaire structure does not obviously increased compared with the SFRB luminaire structure.

\section{Data Availability}

The data used to support the findings of this study are available from the corresponding author upon request. 


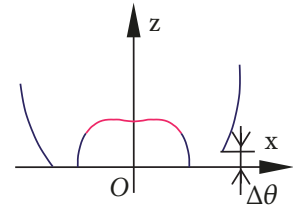

(a)

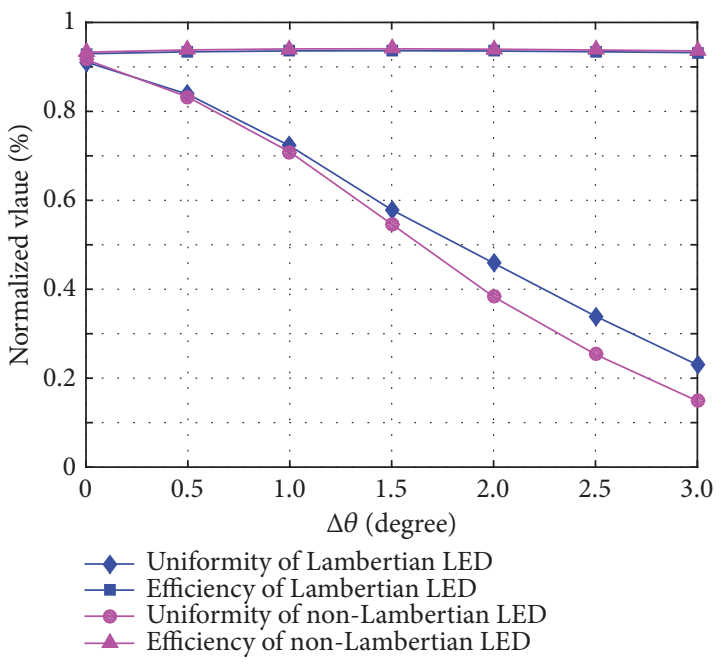

(b)

Figure 10: (a) Schematic of rotational deviation $\Delta \theta$ and (b) influence of rotational deviation on the illumination uniformity and the light utilization efficiency.

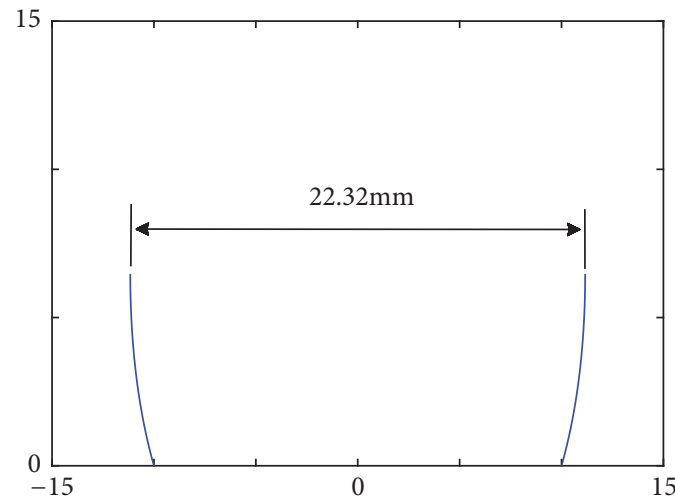

(a)

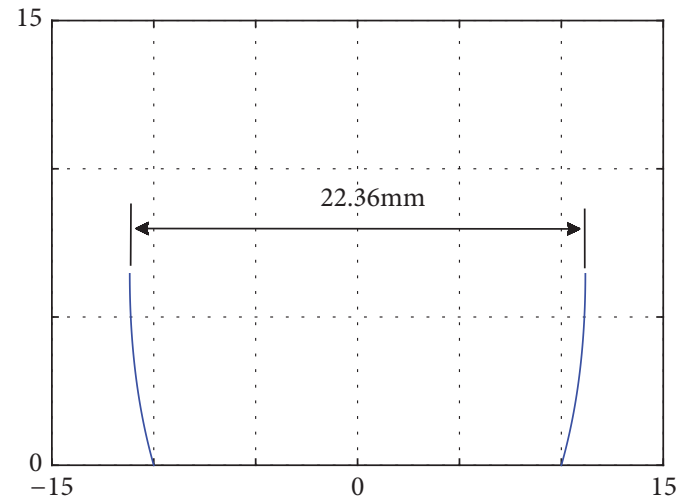

(b)

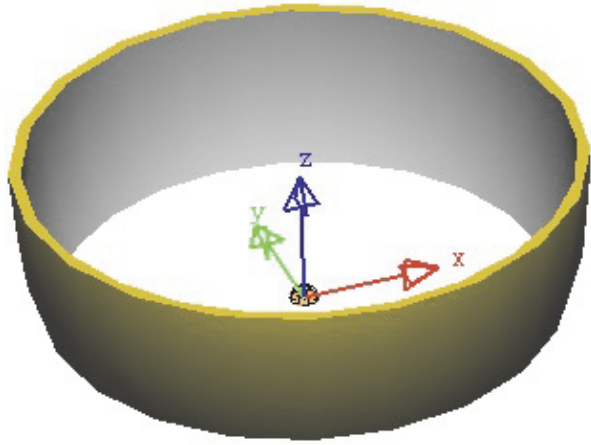

(c)

FIGURE 11: Geometrical profiles of the single freeform reflectors for (a) the ideal Lambertian LED and (b) for non-Lambertian LED and (c) the geometrical model of the freeform reflector for the ideal Lambertian LED. 


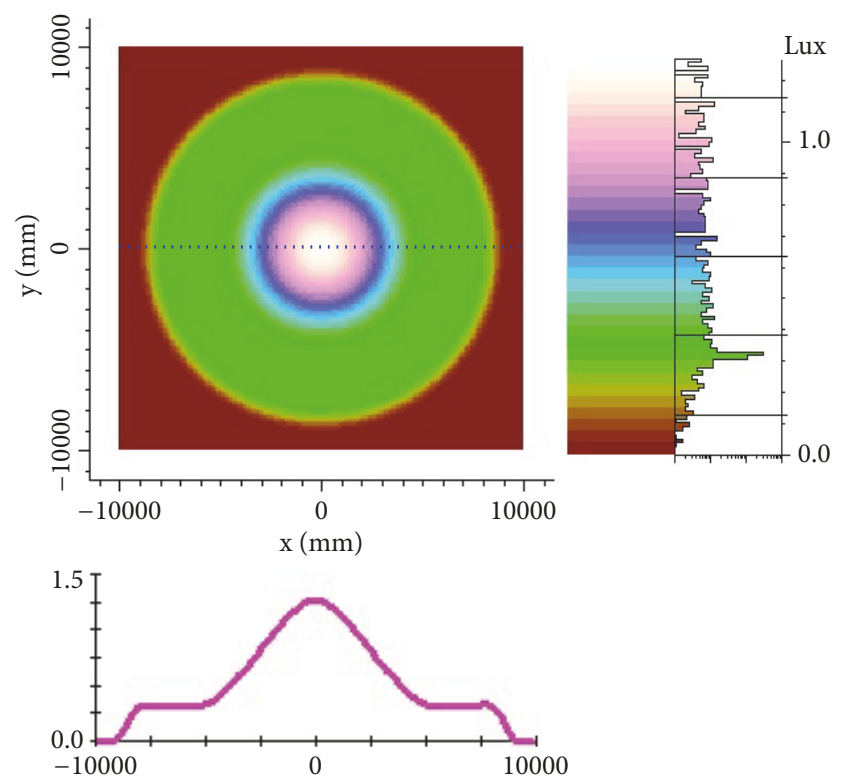

FIGURE 12: Irradiance map of the SFRB lighting unit for the ideal Lambertian LED light source.
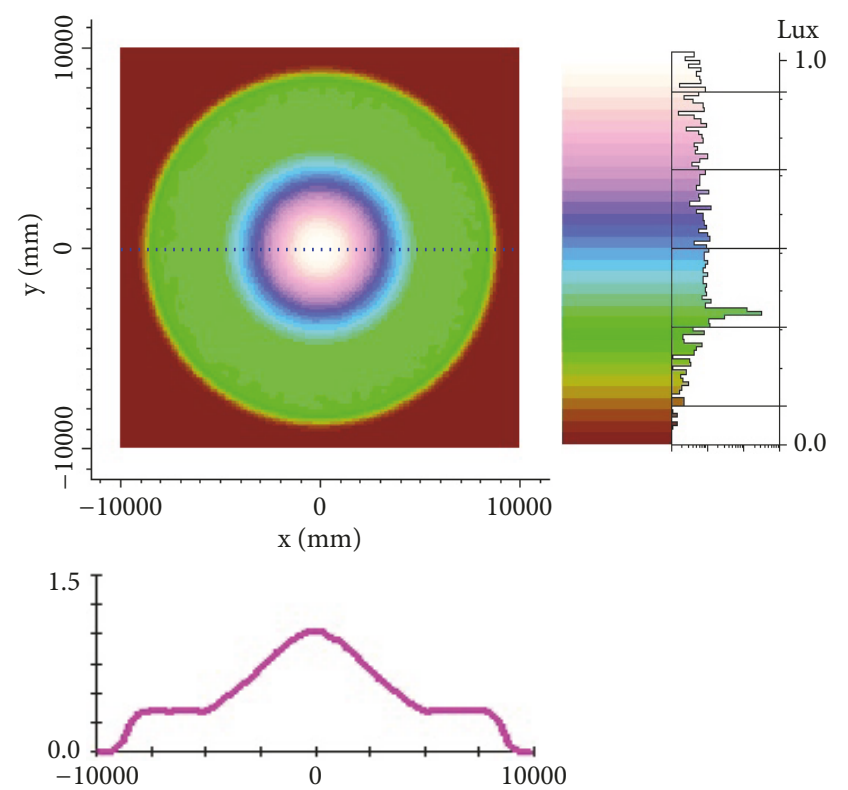

(a)

FIGURE 13: Irradiance map of the SFRB lighting unit for the nonLambertian LED light source.

\section{Conflicts of Interest}

The author declares that there are no conflicts of interest regarding this paper.

\section{Acknowledgments}

The author would like to thank the support of the Scientific Research Fund of Yunnan Open University (no. 18YNOU01).

\section{References}

[1] M. G. Craford, "LEDs for solid state lighting and other emerging applications: status, trends, and challenges," Proc. SPIE, vol. 5941, Article ID 594101, 2005.

[2] M. H. Crawford, "LEDs for solid-state lighting: performance challenges and recent advances," IEEE Journal of Selected Topics in Quantum Electronics, vol. 15, no. 4, pp. 1028-1040, 2009.

[3] X. Long, J. He, J. Zhou et al., "A review on light-emitting diode based automotive headlamps," Renewable \& Sustainable Energy Reviews, vol. 41, pp. 29-41, 2015.

[4] T.-X. Lee and B.-S. Chen, "High Uniformity and Tolerance Design for Direct-Lit LED Backlight Illumination Using Lagrange Interpolation," Journal of Display Technology, vol. 12, no. 11, pp. 1403-1410, 2016.

[5] Z. Zhenrong, H. Xiang, and L. Xu, "Freeform surface lens for LED uniform illumination," Applied Optics, vol. 48, no. 35, pp. 6627-6634, 2009.

[6] A. Gitin, "Designing illuminating devices with faceted reflectors," Optik - International Journal for Light and Electron Optics, vol. 124, no. 22, pp. 5801-5806, 2013.

[7] Z. Zhuang, P. Surman, and F. Yu, "A freeform optics design with limited data for extended LED light sources," Journal of Modern Optics, vol. 63, no. 21, pp. 2151-2158, 2016.

[8] M. A. Moiseev and L. L. Doskolovich, "Design of TIR optics generating the prescribed irradiance distribution in the circle region," Journal of the Optical Society of America A: Optics and Image Science, and Vision, vol. 29, no. 9, pp. 1758-1763, 2012.

[9] J. S. Yang, J.-H. Park, O. Beom-Hoan, S.-G. Park, and S. G. Lee, "Design method for a total internal reflection LED lens with double freeform surfaces for narrow and uniform illumination," Journal of the Optical Society of Korea, vol. 20, no. 5, pp. 614-622, 2016.

[10] X. Zhang and D. Liu, "Freeform lenses for illumination of specific shapes in (u,v) coordinate system," Optical Review, vol. 23, no. 1, pp. 77-83, 2016.

[11] J. Yan, "Design and analysis of reflectors for non-lambertian light-emitting diodes," Optical and Quantum Electronics, vol. 48, no. 12, 2016.

[12] A. Pawlak and K. Zaremba, "Reflector luminaire with high power light-emitting diodes for general lighting," Applied Optics, vol. 47, no. 3, pp. 467-473, 2008.

[13] J.-W. Pan, S.-H. Tu, W.-S. Sun, C.-M. Wang, and J.-Y. Chang, "Integration of non-lambertian LED and reflective optical element as efficient street lamp," Optics Express, vol. 18, no. 102, pp. A221-A230, 2010.

[14] J. Chaves, Introduction to nonimaging optics, vol. 2nd, CRC Press, Boca Raton, Fla, USA, 2015.

[15] S. Wang, K. Wang, F. Chen, and S. Liu, "Design of primary optics for LED chip array in road lighting application," Optics Express, vol. 19, no. 104, pp. A716-A724, 2011.

[16] S. Yu, Y. Tang, Z. Li, Y. Chen, B. Yu, and G. Liang, "Freeform illumination lens design combining energy and intensity mapping," Optical Engineering, vol. 56, no. 4, p. 045101, 2017.

[17] A. Wei, J. R. Sze, and J. L. Chern, "Light-emitting diodes on curved ceramic substrate with primary optics for modification of luminous intensity," Journal of Photonics for Energy, vol. 2, no. 1, Article ID 026501, 2012.

[18] H. Ries and A. Rabl, "Edge-ray principle of nonimaging optics," Journal of the Optical Society of America A: Optics and Image Science, and Vision, vol. 11, no. 10, pp. 2627-2632, 1994. 
[19] J. H. Mathews and K. D. Fink, Numerical Methods Using Matlab, vol. 4th, Pearson Education Inc, 2004.

[20] K. Wang, S. Liu, F. Chen, Z. Qin, Z. Liu, and X. Luo, "Freeform LED lens for rectangularly prescribed illumination," Journal of Optics A: Pure and Applied Optics, vol. 11, no. 10, p. 105501, 2009.

[21] I. Moreno and C.-C. Sun, "Modeling the radiation pattern of LEDs,” Optics Express, vol. 16, no. 3, pp. 1808-1819, 2008. 


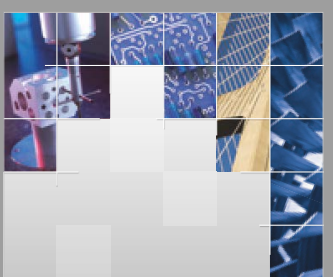

\section{Enfincering}
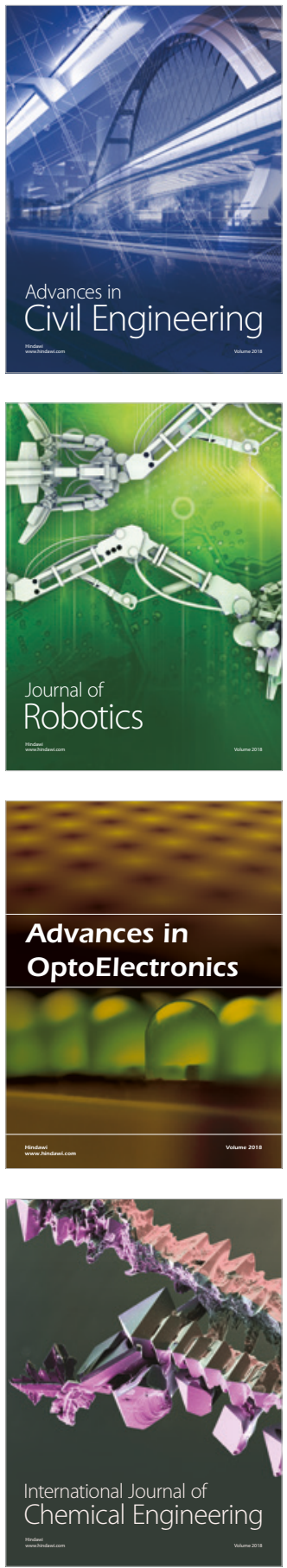

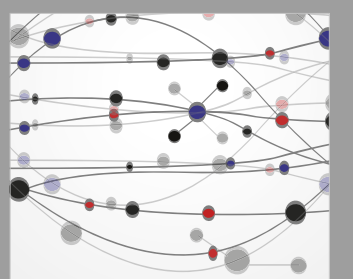

\section{Rotating \\ Machinery}

The Scientific World Journal

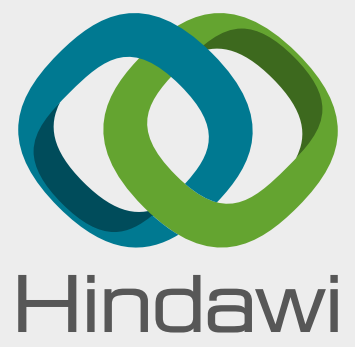

Submit your manuscripts at

www.hindawi.com
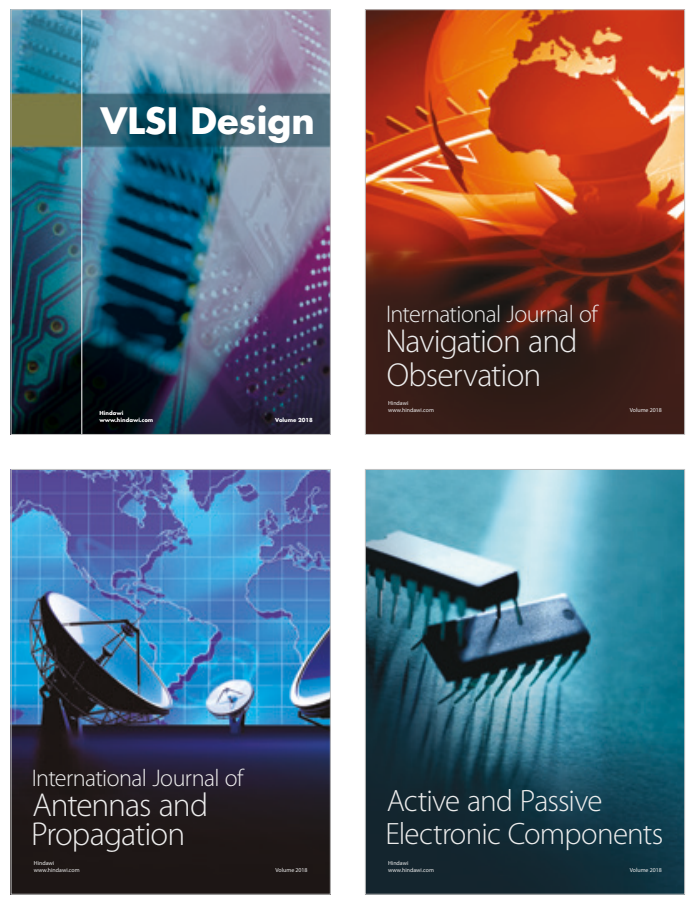
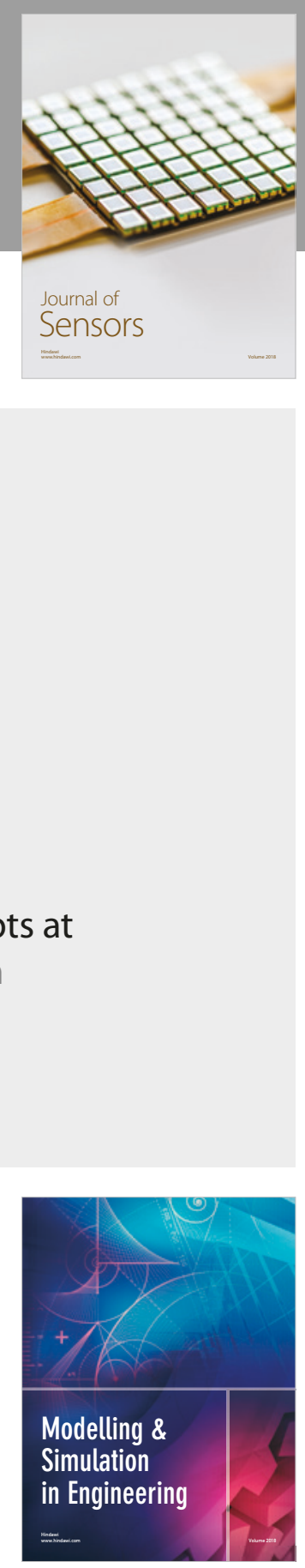

\section{Advances \\ Multimedia}
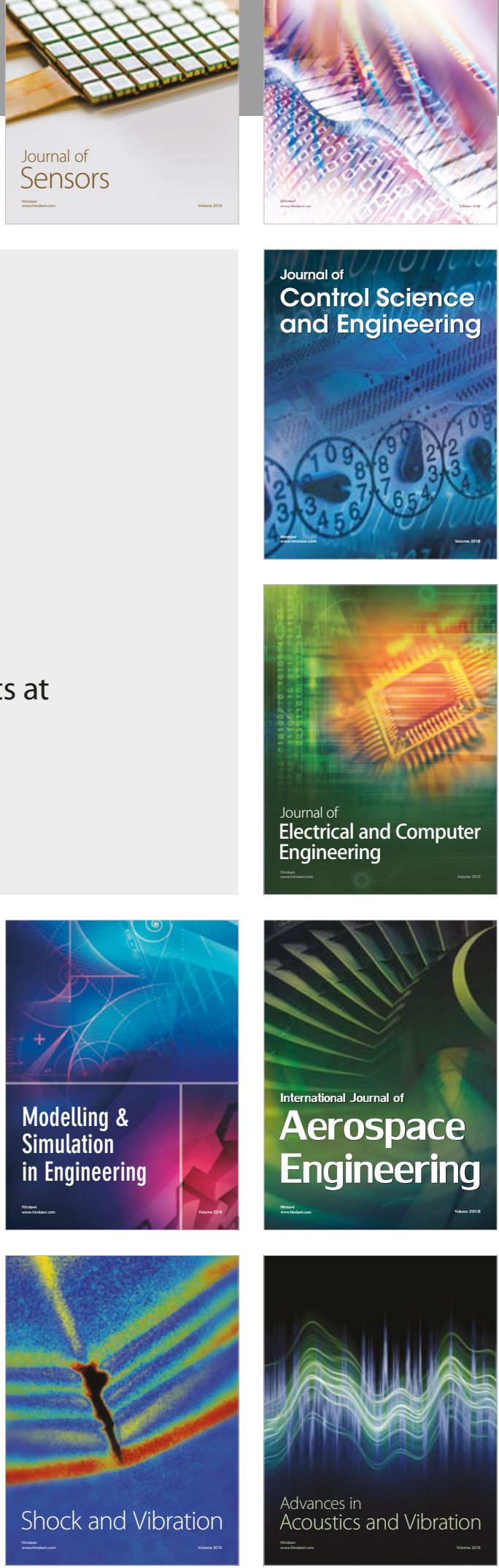